\title{
Progress in the research of GSH in cells
}

\author{
ZHAO HongMei ${ }^{*}$, RUAN HaiHua \& LI HongTao* \\ Tianjin Key Laboratory of Food Biotechnology, College of Biotechnology and Food Science, Tianjin University of Commerce, Tianjin 300134, \\ China
}

Received December 31, 2010; accepted April 22, 2011

\begin{abstract}
Glutathione (GSH), $\gamma$-Glu-Cys-Gly, is one of the most abundant small non-protein thiol molecules in mammalian tissues, particularly in the liver. Although glutathione is present in thiol-reduced (GSH) and disulfide oxidized (GSSG) forms, the predominant form is GSH and its content can exceed $10 \mathrm{mmol} / \mathrm{L}$ in liver cells. As an important intracellular reductant, GSH has many biological functions in cells. Its major function is as an anti-oxidant as it can protect proteins from oxidation by reversible posttranslational modification (glutathionylation) and decrease reactive oxygen species-mediated damage. However, it does have numerous other functions, including to chelate metal irons; enhance the absorption of iron, selenium and calcium; participate in lipid and insulin metabolism; regulate cellular events such as gene expression, DNA and protein synthesis, cell proliferation and apoptosis, redox-dependent signal transduction pathways, cytokine production and the immune response; and control protein glutathionylation. Therefore, GSH plays important roles in cell survival and health, and an imbalance in the GSH level can lead to many diseases. In this review, we provide an overview of the function of GSH in mammalian cells and discuss future research of GSH.
\end{abstract}

glutathione, GSSG, ROS, glutathionylation

Citation: Zhao H M, Ruan H H, Li H T. Progress in the research of GSH in cells. Chinese Sci Bull, 2011, 56: 3057-3063, doi: 10.1007/s11434-011-4689-3

Glutathione (GSH) is the most abundant intracellular nonprotein thiol, and was first identified by Hopkins [1] and Kendall [2]. About $85 \%-90 \%$ GSH is freely distributed in the cytosol, but it is also present in organelles including the mitochondria, peroxisomes, nuclear matrix and endoplasmic reticulum (ER). It is mainly produced in cells to help protect against oxidative stress. It is well known that peroxides are highly unstable and readily form very damaging free radicals upon decomposition, and these radical groups are the main source of oxidative stress. As an important reductant, GSH can scavenge these free radicals using the thiol bridge (i.e., via the sulfhydryl $[-\mathrm{SH}]$ groups), which generates water and yields the oxidized form of glutathione (GSSG).

Via the reducing power of its free sulfhydryl (-SH), GSH plays a key role in many cellular processes. For example, Pan and Berk's research group [3] has shown that glutathionylation regulates TNF- $\alpha$-induced capase- 3 cleavage and apoptosis, and that capase- 3 glutathionylation attenuates

*Corresponding authors (email: hongtaoli@tjcu.edu.cn; wwlovehm@yahoo.com.cn) caspase- 3 cleavage and inhibits endothelial cell death. This research group also showed that protein glutathionylation can regulate the cell death pathway [3]. Because GSH can also detoxify metal irons and ROS, it can participate in protein and DNA synthesis, and affect cell proliferation. In the following review, we will describe the characteristics, functions and future prospects for GSH.

\section{Structure and formation of GSH}

GSH is the most abundant low molecular weight intracellular thiol and is synthesized in most biological organisms. GSH is synthesized from its constituent amino acids by the consecutive action of two enzymes: $\gamma$-glutamyl cysteine ligase $(\gamma$-GCL) and glutathione synthase (GS). The synthesis of GSH occurs intracellularly via a two-stage reaction, with both stages consuming ATP. During $\gamma$-GCL catalysis, the precursor amino acids cysteine and glutamate are combined to form a dipeptide, in a rate-limiting reaction. Next, the 
dipeptide is combined with glycine to form GSH under GS catalysis [4]. GSH can then be recycled via the activity of two enzymes, $\gamma$-glutamyl transpeptidase and dipeptidase, which decomposes GSH into cysteine, glycine and $\gamma$-glutamate.

GSH is present in either a reduced form (GSH) or an oxidized form (GSSG). The balance between GSH and GSSG is associated with the activity of two enzymes: glutathione reductase (GR) and glutathione peroxidase (GPx). During oxidative stress, GSH is oxidized via GPx to form GSSG to alleviate oxidative stress. Conversely, GSSG is reduced to GSH by GR to alleviate reduced stress. Clearly, the balance between GSSG and GSH is important to protect the physiological functions of cells. Figure 1 shows a schematic of GSH recycling and the oxidation/reduction pathways.

Besides the essential enzymes and amino acids, there are many factors that regulate GSH synthesis. The activity of GCL determines the rate of GSH synthesis and ultimately affects GSH synthesis. GCL comprises two subunits, a catalytic subunit (GCLC) and a modifier subunit (GCLM). Several factors regulate GCLC activity including NF-E2 related factor 2 (Nrf2), transforming growth factor- $\beta 1$ (TGF- $\beta 1$ ) and hepatocyte growth factor (HGF). Upon recognition of the signals imparted by oxidized stress, Nrf2 is released from keap1, escapes from proteosomal degradation, and translocates to the nucleus to induce GCL gene expression [5]. Interestingly, Nrf1/Nrf2-knockout mice have lower GCLC expression than wild-type mice [6,7]. TGF- $\beta 1$ is a pleiotropic cytokine elevated in many chronic inflam- matory disorders that can inhibit GCLC expression and negatively regulate GSH synthesis [8]. HGF is a mitogen that induces the expression of both GCL subunits, although the low density unit induced the expression of GCLC only and increased GSH levels in subconfluent cells but not in confluent cells [9].

In addition to the above findings, stabilization of GCLC mRNA and phosphorylation of GCLC by protein kinase A, protein kinase $\mathrm{C}$ and $\mathrm{Ca}^{2+}$-calmodulin kinase II are known to regulate GSH synthesis [10]. Another post-translational regulatory pathway of GCLC involves a direct target for caspase 3-mediated cleavage that occurs at $\mathrm{Asp}^{499}$ in the sequence $\mathrm{AVVD}^{499} \mathrm{G}$, a target involved in some forms of apoptotic cell death. In turn, cleaved GCLC decreases GSH synthesis [11]. AP- 1 and activation of NF- $\kappa$ B by TNF- $\alpha$ are also involved in the regulation of GSH [12]. Meanwhile, many physiological and biochemical studies in mammalian tissues and micro-organisms have shown that GSH can regulate its own formation by acting on the $\gamma$-GCL. In brief, many regulators participate in GSH synthesis.

Considering the number of GSH transferases expressed in cells, GSH can conjugate to a large number of endogenous compounds to perform its physiologic and metabolic functions. These targets of GSH include 1-chloro-2,4dinitrobenzene; benzo(a)pyrene-4-5-oxide; cholesterol-5 $\alpha$, $6 \alpha$-oxide; benzo(a)pyrene-7,8-diol-9,10-oxide and aflatoxin B1 [13], as well as reactive oxygen species (ROS), toxic metals and the cysteine residue of proteins.

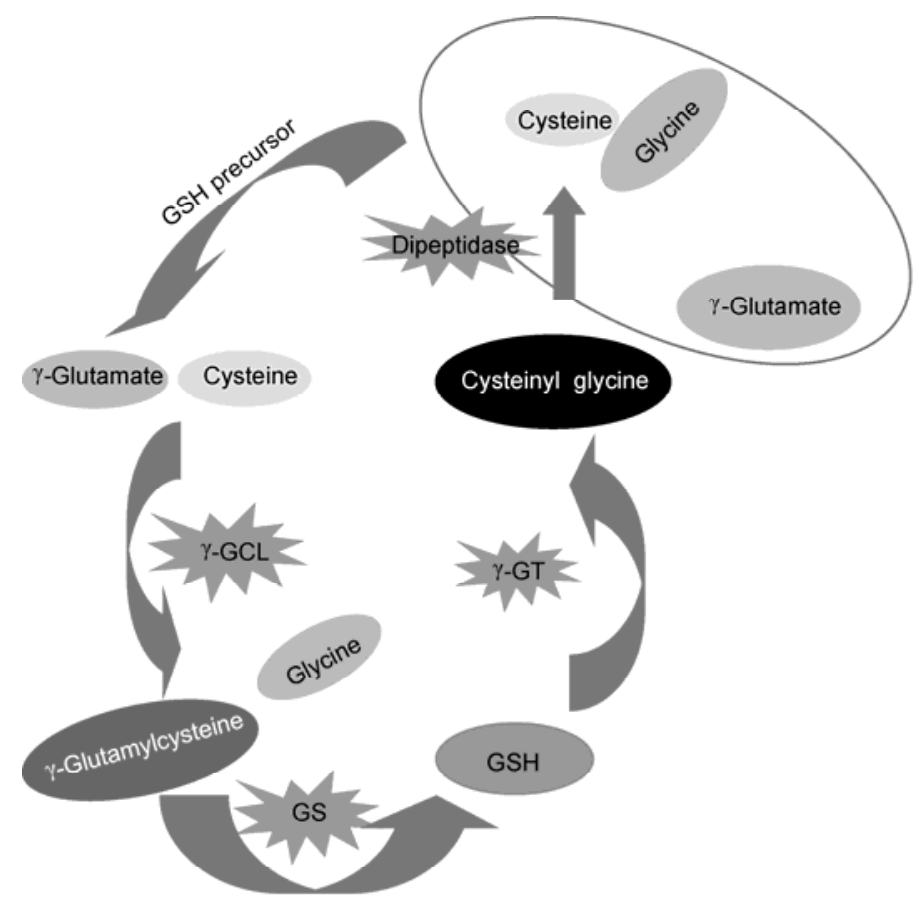

(a)<smiles>NC(CCC(=O)NC(S)C(=O)NCC(=O)O)C(=O)O</smiles>

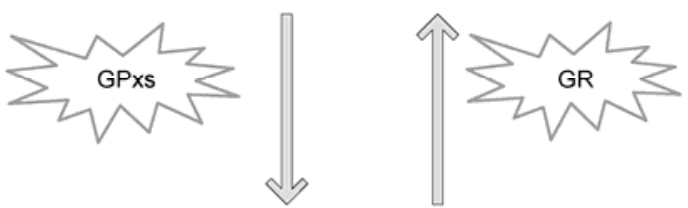<smiles>NC(CCC(=O)NC(SSC(NC(=O)CCC(N)C(=O)O)C(=O)NCC(=O)O)C(=O)NCC(=O)O)C(=O)O</smiles>

(b)

Figure 1 (a) GSH synthesis and recycling in cells; (b) the conversion of GSH and GSSG. 


\section{Function of GSH in cells}

\subsection{GSH is an antioxidant that protects against radical damage}

The level of GSH is a major indicator of oxidative stress. ROS, such as $\mathrm{H}_{2} \mathrm{O}_{2}, \mathrm{OH} \cdot$, ONOO $\cdot$ and $\mathrm{O}_{2} \cdot$, are the main radicals that cause cellular damage by oxidation of cell membrane lipids and cytosolic proteins, which ultimately affects cell signaling. Reduced GSH is the main ROS scavenger that protects cells from oxidative damage by the antioxidant activity of its free thiol residue.

The cysteine thiol residues of proteins are exceptionally useful in terms of structural and regulatory aspects, but are also exceptionally vulnerable to oxidation. Conserved cysteines are highly important for the function of metabolic enzymes and for signaling processes underlying the responses to environmental factors. If the cysteines are oxidized by ROS, their structural, catalytic and/or regulatory functions may be affected [14]. GSH can directly detoxify ROS via a reaction catalyzed by GPx and hence protect the cysteine residues.

\subsection{GSH can detoxify cytotoxic molecules}

Although GSH is one of the most important antioxidants in mammalian cells, it also plays a key role in the detoxification of electrophilic compounds, including ROS free radicals, toxic metals (e.g., selenium, zinc, chromium, mercury, cadmium, arsenic, silver and lead) [15] and microbe effector proteins. These toxic materials can induce many diseases. As a scavenger, GSH can detoxify these cytotoxic molecules through its thiol group. Of note, GSH contain six possible metal binding sites [15], and can bind to the metals to stabilize them in a non-reactive form or to form different species by redox reactions. Accordingly, GSH decreases the toxicity of metals.

Through the Michael addition reaction or disulfide formation, the GSH thiol decreases the effects of toxic compounds or proteins. For example, ROS can easily oxidize lipids to generate large quantities of highly reactive $\alpha, \beta$ unsaturated aldehydes (enals). At low concentrations, enals such as 4-hydroxy trans-2-nonenal can affect cell signal transduction while high concentrations of enals are cytotoxic. The GSH thiol can adduct the unsaturated bond of the enals to reduce the cytotoxic effects [16]. Thus, the conjugation of reactive drug metabolites to GSH is considered to be an important detoxification mechanism, GSH can also decrease xenobiotic toxicity in erythrocytes in this manner [17], while the toxicity of polycyclic aromatic hydrocarbons, a known carcinogen, can also be reduced by GSH-mediated detoxification as GSH can adduct the bay- and fjord-region diol epoxides for detoxification [17-19]. The aflatoxin $\mathrm{B}(1)-8,9$-epoxide is a hepatocarcinogenic intermediate of aflatoxin $\mathrm{B}(1)$ that can be detoxified through GSH conjuga- tion catalyzed by GSTA3 [20]. Finally, heterocyclic amines derived from food, particularly 2-amino-1-methyl-6phenylimidazo[4,5-b]pyridine ( $\mathrm{PhIP}$ ), are involved in the etiology of human colorectal cancer as $N$-oxidized PhIP react with DNA. Notably, the toxicity of $N$-acetoxy-PhIP can be decreased by conjugation with GSH in a reaction catalyzed by GSTA1-1 [21].

\subsection{Signal transduction}

It is well known that ROS function as redox messengers in intracellular signal transduction and regulation and that GSH is the main scavenger of ROS. Apart from its antioxidant and detoxification activities, GSH also plays important roles in signaling processes and in the crosstalk of the redox signal with other signaling pathways. GSH regulates signal transduction pathways, while some signal transducers regulate the GSH level. We will discuss the activities of GSH in terms of the regulation of signal transduction below.

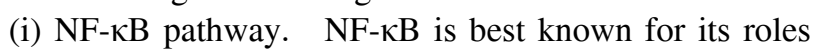
in immune homeostasis and inflammation, while it has another important role in the regulation of the redox signaling pathway and is regulated by GSH. Meng et al. [22] reported that constitutive NF- $\kappa \mathrm{B}$ activity in cancer cells can enhance GSH synthesis, while inhibition of NF- $\mathrm{BB}$ activity decreases GSH levels. Loss of the IKK- $\beta$ signal significantly reduced basal NF- $\kappa B$ activity and decreased the binding of NF- $\mathrm{KB}$ to the promoters of GCLC and GCLM, reducing the expression of both subunits. Thus, because GCLC and GCLM expression is directly related to GSH biosynthesis, NF- $\mathrm{kB}$ indirectly affects GSH synthesis [23].

Recently, we have found some data to indicate that GSH or glutathionylation of proteins by GSH can also affect NF- $\mathrm{KB}$ activity. For example, glutathionylation of p65 may be responsible for cinnamaldehyde-mediated NF- $\mathrm{\kappa B}$ inactivation [24] while redox regulation by glutaredoxin in retinal glial cells is disturbed by hyperglycemia, leading to NF- $\mathrm{BB}$ activation and a pro-inflammatory response [25].

(ii) Death pathway. Recent studies have suggested that GSH depletion and post-translational modification of proteins through glutathionylation are related to cell death. GSH is crucial to counteract oxidative stress-induced liver injury as it detoxifies some hepatotoxins and inhibits cell damage induced by hepatotoxins (e.g., Flavokawain B, pyrrolizidine alkaloid, aflatoxin B1 and ethanol) [26-28]. In addition to hepatotoxins, GSH is recognized by compounds such as gallic acid, which is widely distributed in plants and foods, and can induce Hela cell death in relation to the ROS and GSH level [29]. Depletion of mitochondrial GSH in the human B lymphoma cell line by treatment with $L$-buthionine sulfoximine induced caspase- 3 cleavage and apoptosis [30] indicating that GSH may be a potent early activator of the apoptotic signal.

(iii) Cytokine synthesis. The glutathione-redox balance, represented by the ratio of GSH to GSSG, is an important 
regulator of cellular immune responses by participating in cytokine synthesis. Intestinal subepithelial myofibroblasts from Crohn's disease patients exhibited an increased oxidative state because of a decrease in the GSH/GSSG ratio, which was related to an increase in IL-6 production [31]. The change in the glutathione-redox balance in macrophages by the activity of GSH donors (e.g., reduced glutathione ethyl ester or $N$-acetyl- $L$-cysteine) can differentially regulate the production of IL-12. At low concentrations, $N$-acetyl- $L$-cysteine increased IL-12 p40/p70 production. In contrast, at high concentrations, $N$-acetyl- $L$-cysteine reduced IL-12 production because of increased calmodulin expression [32].

\subsection{Metabolism}

GSH can protect lipids, protein and small molecules from oxidized damage and may play a vital role in metabolism. Unfortunately, the precise role of GSH in metabolism is still unknown as studies have only reported the effects of GSH changes on metabolic imbalances. For example, a decrease in GSH accelerates adipogenesis in pre-adipocytes [33]. Meanwhile, oxidative stress is related to several metabolic abnormalities observed in obesity [34]. Glyoxalase 1 (Glo1) and glyoxalase 2 (Glo2) are enzymes that catalyze the conversion of toxic a-oxo-aldehydes into the corresponding a-hydroxyl acids using $L$-GSH as a cofactor and glutathionylation of Glo1 on cysteine 139 is an important posttranslational modification, which strongly inhibits enzyme activity. Thus, inhibition of Glo1 is expected to be an important factor within the context of redox-dependent regulation of glucose metabolism in cells [35].

Learning from the metabolic phenomena associated with varying levels of GSH, we speculate that GSH may be involved in many components of metabolism by glutathionylation of crucial metabolism enzymes or formation of disulfide bridges to the change redox balance and hence affect metabolism.

\subsection{Cell cycle}

GSH is abundant in cells and is found in most cellular compartments, including the nucleus. GSH plays a pivotal role in cell proliferation by its transport between the different intracellular compartments. Analyses by many researchers have concluded that GSH affect the cell cycle through different mechanisms. Some researchers have found that GSH co-localizes with nuclear DNA at the early stage of proliferation in plant and animal cells [36]. During the G1 and S phases of the cell cycle, GSH aggregation and sequestration in the nucleus has a marked effect on cellular redox homoeostasis and gene expression. When GSH is sequestered in the nucleus, the levels of transcripts encoding stress and defense proteins are decreased. It can also regulate the activity of telomerase in vivo, an enzyme that is coordinated with changes in the critical cell cycle proteins, particularly id2 and E2F4 [37]. Other than its effects on gene expression by regulating histones and PARP, GSH can also enable the cell to retain memory of the cellular redox environment [38].

Overall, GSH participates in many biochemical reactions in cells and maintains the redox balance to maintain normal cellular activity. We have summarized the intracellular roles of GSH in Figure 2.

\section{GSH-related diseases}

Because GSH plays a central role in regulating the redox pathway, changes in the level of GSH can induce some changes in physiologic indicators and may potentially induce certain diseases. For example, recent studies have shown that elderly people with hypertension have higher GSH concentrations, higher activities of GSH transferase and GSH reductase in erythrocytes, but no difference in GSH peroxidase activity, indicating that GSH play some role in the regulation of blood pressure [39]. Chon et al. [40] used GSH-depleted mice to show that differential expression of GSH affects energy and protein metabolism in relation to the pathogenetic background of hypertension. Staron et al. [41] found that rheumatoid arthritis (RA) patients have higher ROS levels than healthy people, whereas the expression of the antioxidant enzymes catalase and GPx were not significant different, and that superoxide dismutase antioxidant activity and - $\mathrm{SH}$ levels were lower in RA patients than in the control group. The GSH:GSSG ratio was significantly $(P<0.05)$ decreased in the plasma and lymphocyte cells of patients with Ménière's disease [42].

Many neurodegenerative disorders, including amyotrophic lateral sclerosis, Alzheimer's disease, and Parkinson's disease, are associated with oxidative stress and the failure of antioxidant systems, particularly a decrease in GSH content $[43,44]$.

Infection diseases are also associated with concomitant changes in GSH levels. For example, patients with Helicobacter pylori-infected gastric epithelial cells had significantly lower concentrations of GSH than normal controls, and that the reduced GSH levels following $H$. pylori colonization of the gastric mucosa may be caused by a direct effect of the bacterium and by associated inflammatory responses [45]. Pyocyanin extracted from Pseudomonas aeruginosa bacteria affect human bronchial epithelial (HBE) cells in the following way: mitochondrial GSSG is increased, oxidant-sensitive proteins (HIF-1 $\alpha$ and HO-1) are increased, and GSH is decreased. Changes in thiol levels as well as oxidant-sensitive proteins may contribute to $P$. aeruginosa-mediated lung injury [46]. As infection with $H$. pylori and $P$. aeruginosa reduce the host's GSH levels, GSH may offer an attractive therapy target in the future. In fact, some researchers have been trying to target GSH to 


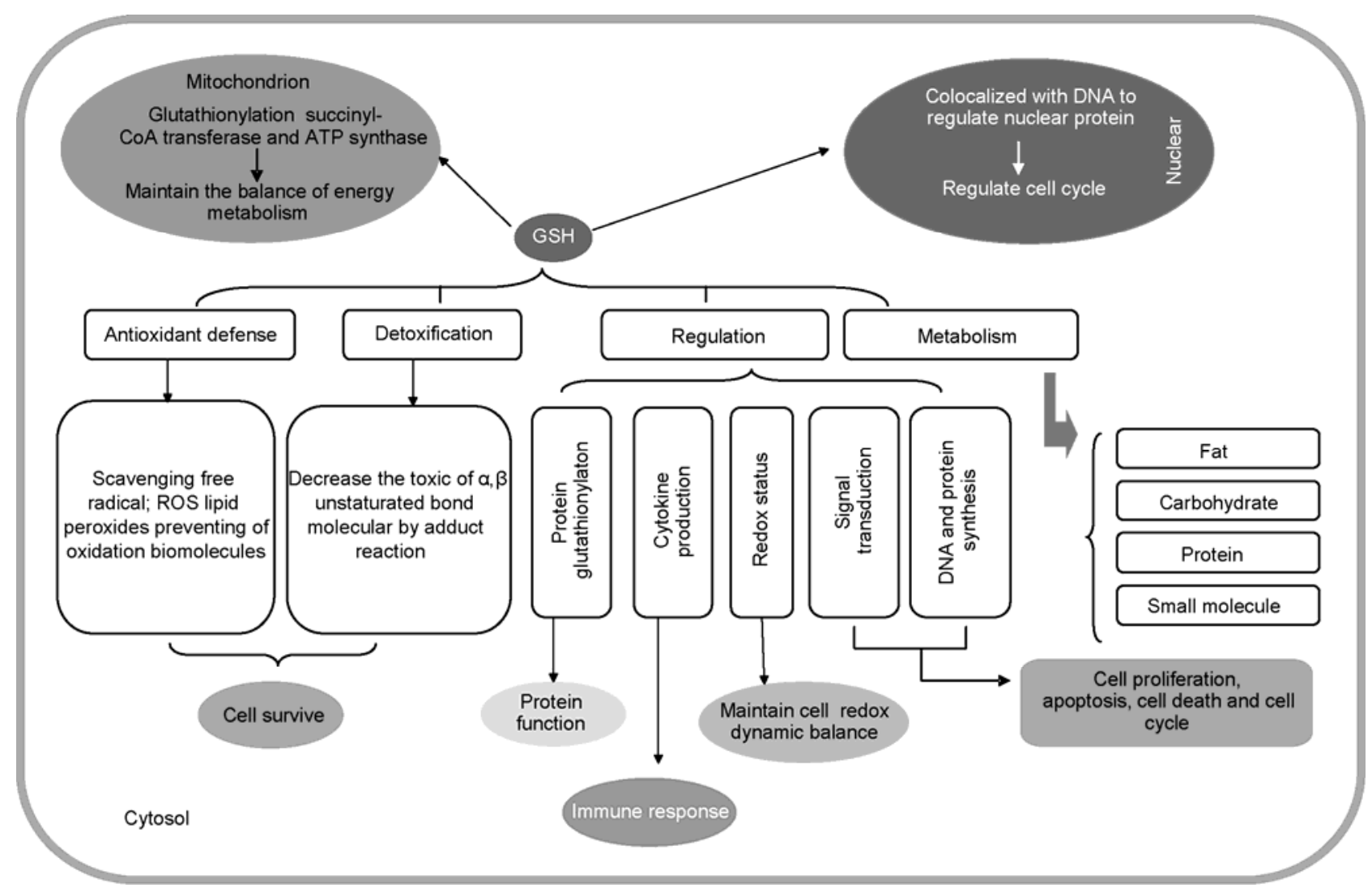

Figure 2 Intracellular roles of GSH. In the mitochondrion, GSH can regulate succinyl-CoA transferase and ATP synthase to maintain energy metabolism. In the nucleus, GSH regulates the cell cycle by co-localizing with DNA to regulate nuclear protein expression. In the cytosol, GSH has many roles, including antioxidant defense, detoxification, protein synthesis and modification and maintain the redox balance. In addition, GSH also participates in many metabolic processing of many biological materials.

protect against infection. For example, $L$-cysteine can increase GSH synthesis and the subsequent increases in GSH levels can enhance the defense against influenza virus infection [47].

We have learned from these findings that a decrease in GSH can lead to many diseases, benefit pathogen infection and have adverse effects on the host. Therefore maintaining the GSH balance in cells is very important and necessary to maintain the health of cells and organisms.

\section{Concluding remarks and future perspectives for GSH research}

GSH takes part in many biochemical reactions in cells through its thiol group by forming adducts with unsaturated bonds or forming disulfide bonds with proteins containing cysteine. Although many studies of GSH and glutathionylation have been conducted, they have consistently described phenomena, while the mechanisms involved in the activity of GSH and glutathionylation remain largely unknown.

Many diseases and some pathogen infections affect GSH levels. Most pathogens increase ROS levels and decrease GSH levels. Therefore, some researchers have used ROS or
GSH as therapeutic targets. Antioxidants such as $N$-acetylcysteine, $S$-acetyl glutathione and $S$-acetyl-beta-mercaptoethylamine can replenish the depleted GSH levels during infection and inhibit pathogen invasion. Furthermore, these small molecules are considered to be pro-drugs that can inhibit complications of HIV [48]. Although we know that infection with many pathogens can affect GSH levels, the mechanisms responsible for changes in GSH levels and how the changes in GSH levels affect the cell response are unclear.

In conclusion, GSH plays many extremely important roles in maintaining the health of cells, and may offer a therapeutic target for some diseases. Therefore, we believe it is important to further explore the functions of GSH. Although much research has been published on GSH, these studies have mainly described the phenomena and speculated on mechanisms; the real function and mechanism of GSH remain unknown, and we must endeavor to do our best to solve this mystery.

This work was supported by the National Natural Science Foundation of China (30970630), the New Century Talents Program (NCET-08-0912) and the Key Project of the Chinese Ministry of Education (210010). 
1 Hopkins F G. Glutathione: Its influence in oxidation of fats and proteins. Biochem J, 1925, 19: 787-819

2 Kendall E C, Loewen D F. The mechanism of oxidation-reduction potential: The oxidation-reduction potential of cysteine and cystine. Biochem J, 1928, 22: 669-682

3 Pan S, Berk B C. Glutathiolation regulates tumor necrosis factoralpha-induced caspase-3 cleavage and apoptosis: Key role for glutaredoxin in the death pathway. Circ Res, 2007, 100: 152-154

4 Deneke S M, Fanburg B L. Regulation of cellular glutathione. Am J Physiol, 1989, 257: 163-173

5 Kensler T W, Wakabayashi N, Biswal S. Cell survival responses to environmental stresses via the Keap1-Nrf2-ARE pathway. Annu Rev Pharmacol Toxicol, 2007, 47: 89-116

6 Chan K, Han X D, Kan Y W. An important function of Nrf2 in combating oxidative stress: Detoxification of acetaminophen. Proc Natl Acad Sci USA, 2001, 98: 4611-4616

7 Chen L, Kwong M, Lu R, et al. Nrf1 is critical for redox balance and survival of liver cells during development. Mol Cell Biol, 2003, 23: 4673-4686

8 Franklin C C, Rosenfled-Franklin M E, White C, et al. TGF $\beta 1$ induced suppression of glutathione antioxidant defenses in hepatocytes: Caspase-dependent posttranslational and caspase independent transcriptional regulatory mechanisms. FASEB J, 2003, 17: 1535-1537

9 Yang H P, Magilnick N, Xia M, et al. Effects of hepatocyte growth factor on glutathione synthesis, growth, and apoptosis is cell densitydependent. Exp Cell Res, 2008, 314: 398-412

10 Franklin C C, Backos D S, Molar I, et al. Structure, function and post-translational regulation of the catalytic and modifier subunits of glutamate cysteine ligase. Mol Aspects Med, 2010, 30: 86-98

11 Franklin C C, Krejsa C M, Pierce R H, et al. Caspase-3 dependent cleavage of the glutamate- $L$-cysteine ligase catalytic subunit during apoptotic cell death. Am J Pathol, 2002, 160: 1887-1894

12 Yang H P, Zeng Y, Lee T D, et al. Role of AP-1 in the coordinate induction of rat glutamate-cysteine ligase and glutathione synthetase by tert-butylhydroquinone. J Biol Chem, 2002, 277: 35232-35239

13 Hiratskuka A, Tobita K, Saito H, et al. (s)-Preferential detoxification of 4-hydroxy-2(E)-nonenal enantiomers by hepatic glutathione $S$-transferase isoforms in guinea-pigs and rats. Bichem J, 2001, 355: 237-244

14 Deneke S M, Fanburg B L. Regulation of cellular glutathione. Am J Physiol, 1989, 257: 163-173

15 Helbig K, Bleuel C, Krauss G J, et al. Glutathione and transition-metal homeostasis in Escherichia coli. J Bacteriol, 2008, 190: 5431-5438

16 Srivastava S, Ramana K V, Bhatnagar A, et al. Synthesis, quantification, characterization, and signaling properties of glutathionyl conjugates of enals. Methods Enzymol, 2010, 474: 297-313

17 Lin T, Yang M S. Benzo[a]pyrene-induced elevation of GSH level protects against oxidative stress and enhances xenobiotic detoxification in human HepG2 cells. Toxicology, 2007, 235: 1-10

18 Gelhaus S L, Harvey R G, Penning T M, et al. Regulation of benzo[a]pyrene-mediated DNA- and glutathione-adduct formation by 2,3,7,8-tetrachlorodibenzo-p-dioxin in human lung cells. Chem Res Toxicol, 2010, 24: 89-98

19 Sundberg K, Dreij K, Seidel A, et al. Glutathione conjugation and DNA adduct formation of dibenzo[a,1]pyrene and benzo[a]pyrene diol epoxides in V79 cells stably expressing different human glutathione transferases. Chem Res Toxicol, 2002, 15: 170-179

20 Gao S S, Chen X Y, Zhu R Z, et al. Sulforaphane induces glutathione S-transferase isozymes which detoxify aflatoxin B (1)-8,9-epoxide in AML 12 cells. Biofactors, 2010, 36: 289-296

21 Coles B, Nowell S A, MacLeod S L, et al. The role of human glutathione $S$-transferases (hGSTs) in the detoxification of the foodderived carcinogen metabolite $N$-acetoxy-PhIP, and the effect of a polymorphism in hGSTA1 on colorectal cancer risk. Mutat Res, 2001, 482: 3-10

22 Meng Q, Peng Z, Chen L, et al. Nuclear factor- $\kappa$ B modulates cellular glutathione and prevents oxidative stress in cancer cells. Cancer Lett, 2010, 299: 45-53
23 Peng Z, Geh E, Chen L, et al. Inhibitor of kappaB kinase beta regulates redox homeostasis by controlling the constitutive levels of glutathione. Mol Pharmacol, 2010, 77: 784-792

24 Liao B C, Hsieh C W, Lin Y C, et al. The glutaredoxin/glutathione system modulates NF-kappaB activity by glutathionylation of p65 in cinnamaldehyde-treated endothelial cells. Toxicol Sci, 2010, 116: 151-163

25 Shelton M D, Kern T S, Mieyal J J. Glutaredoxin regulates nuclear factor kappa-B and intercellular adhesion molecule in Muller cells: Model of diabetic retinopathy. J Biol Chem, 2007, 282: 12467-12474

26 Zhou P, Gross S, Liu J H, et al. Flavokawain B, the hepatotoxic constituent from kava root, induces GSH-sensitive oxidative stress through modulation of IKK/NF-kappaB and MAPK signaling pathways. FASEB J, 2010, 24: 4722-4732

27 Liang Q, Sheng Y, Jiang P, et al. The gender-dependent difference of liver GSH antioxidant system in mice and its influence on isolineinduced liver injury. Toxicology, 2011, 280: 61-69

28 Adedara I A, Owumi S E, Uwaifo A O, et al. Aflatoxin $\mathrm{B}_{1}$ and ethanol co-exposure induces hepatic oxidative damage in mice. Toxicol Ind Health, 2010, 26: 717-724

29 You B R, Park W H. The effects of mitogen-activated protein kinase inhibitors or small interfering RNAs on gallic acid-induced HeLa cell death in relation to reactive oxygen species and glutathione. J Agric Food Chem, 2011, 59: 763-771

30 Armstrong J S, Steinauer K K, Hornung B, et al. Role of glutathione depletion and reactive oxygen species generation in apoptotic signaling in a human B lymphoma cell line. Cell Death Differ, 2002, 9: 252-263

31 Catarzi S, Favilli F, Romagnoli C, et al. Oxidative state and IL-6 production in intestinal myofibroblasts of Crohn's disease patients. Inflamm Bowel Dis, 2011, 17: 1674-1684

32 Alam K, Ghousunnissa S, Nair S, et al. Glutathione-redox balance regulates c-rel-driven IL-12 production in macrophages: Possible implications in antituberculosis immunotherapy. J Immunol, 2010, 184: 2918-2929

33 Vigilanza P, Aquilano K, Baldelli S, et al. Modulation of intracellular glutathione affects adipogenesis in 3T3-L1 cells. J Cell Physiol, 2011, 226: 2016-2024

34 Di Renzo L, Galvano F, Orlandi C, et al. Oxidative stress in normal-weight obese syndrome. Obesity, 2010, 18: 2125-2130

35 Birkenmeier G, Stegemann C, Hoffmann R, et al. Posttranslational modification of human glyoxalase 1 indicates redox-dependent regulation. PLoS ONE, 2010, 5: 2-13

36 Vivancos P D, Dong Y P, Ziegler K, et al. Recruitment of glutathione into the nucleus during cell proliferation adjusts whole-cell redox homeostasis in Arabidopsis thaliana and lowers the oxidative defence shield. Plant J, 2010, 64: 825-838

37 Pallardó F V, Markovic J, García J L, et al. Role of nuclear glutathione as a key regulator of cell proliferation. Mol Aspects Med, 2009, 30: 77-85

38 Diaz V P, Wolff T, Markovic J, et al. A nuclear glutathione cycle within the cell cycle. Biochem J, 2010, 431: 169-178

39 Rybka J, Kupczyk D, Kędziora-Kornatowska K, et al. Glutathione-related antioxidant defense system in elderly patients treated for hypertension. Cardiovasc Toxicol, 2011, 11: 1-9

40 Chon H, Bluyssen H A R, Holstege F C P, et al. Gene expression of energy and protein metabolism in hearts of hypertensive nitric oxideor GSH-depleted mice. Eur J Pharmacol, 2005, 513: 21-33

41 Staroń A, Mąkosa G, Koter-Michalak M. Oxidative stress in erythrocytes from patients with rheumatoid arthritis. Rheumatol Int, 2010, doi: 10.1007/s00296-010-1611-2

42 Calabrese V, Cornelius C, Maiolino L, et al. Oxidative stress, redox homeostasis and cellular stress response in Ménière's disease: Role of vitagenes. Neurochem Res, 2010, 35: 2208-2217

43 Limón-Pacheco $\mathrm{J} \mathrm{H}$, Gonsebatt $\mathrm{M}$ E. The glutathione system and its regulation by neurohormone melatonin in the central nervous system. Cent Nerv Syst Agents Med Chem, 2010, 10: 287-297

44 Jomova K, Vondrakova D, Lawson M, et al. Metals, oxidative stress and neurodegenerative disorders. Mol Cell Biochem, 2010, 345: 
91-104

45 Shirin H, Pinto J T, Liu L U, et al. Helicobacter pylori decreases gastric mucosal glutathione. Cancer Lett, 2001, 164: 127-133

46 Ahmad I M, Britigan B E, Abdalla M Y. Oxidation of thiols and modification of redox-sensitive signaling in human lung epithelial cells exposed to pseudomonas pyocyanin. $\mathbf{J}$ Toxicol Environ Health A, 2011, 74: 43-51
47 Takagi Y, Kurihara S, Higashi N, et al. Combined administration of $L$-cystine and $L$-theanine enhances immune functions and protects against influenza virus infection in aged mice. J Vet Med Sci, 2010, 72: $157-165$

48 Fraternale A, Paoletti M F, Casabianca A, et al. Inhibition of murine AIDS by pro-glutathione (GSH) molecules. Antiviral Res, 2008, 77 : $120-127$

Open Access This article is distributed under the terms of the Creative Commons Attribution License which permits any use, distribution, and reproduction in any medium, provided the original author(s) and source are credited. 